\title{
Stage I Jejunal Neuroendocrine Tumor AJCC v8
}

National Cancer Institute

\section{Source}

National Cancer Institute. Stage I Jejunal Neuroendocrine Tumor AJCC v8. NCI Thesaurus. Code C135120.

Stage I includes: T1, N0, M0. T1: Tumor invading lamina propria or submucosa and measuring $1 \mathrm{~cm}$ or less in size. N0: No regional lymph node metastasis. M0: No distant metastasis. (AJCC 8th ed.) 